\title{
A Comparative Study on the Wind Deflection of Railway Overhead Contact Line Based on Empirical Formula and Finite Element Approach
}

\author{
Wenping Chu, ${ }^{1}$ Yang Song $\mathbb{D}^{2},{ }^{2}$ and Zhigang Liu ${ }^{1}$ \\ ${ }^{1}$ National Rail Transit Electrification and Automation Engineering Technique Research Centre, Southwest Jiaotong University, \\ Chengdu 610031, China \\ ${ }^{2}$ Department of Structural Engineering, Norwegian University of Science and Technology, Trondheim 7491, Norway
}

Correspondence should be addressed to Yang Song; yang.song@ntnu.no

Received 29 April 2021; Accepted 1 July 2021; Published 12 July 2021

Academic Editor: Edoardo Sabbioni

Copyright (C) 2021 Wenping Chu et al. This is an open access article distributed under the Creative Commons Attribution License, which permits unrestricted use, distribution, and reproduction in any medium, provided the original work is properly cited.

The assessment of wind deflection of the railway overhead contact line (OCL) is of great importance to ensure the safe and reliable operation of the electrified railway. This paper performs a comparative study of the railway OCL wind deflection based on the traditional empirical formula and finite element method (FEM). Considering the geometrical nonlinearity, a nonlinear finite element approach is adopted to model the OCL. The crosswind load is applied to the OCL through an iterative procedure. The comparative study indicates that the maximum wind deflection estimated by the empirical formula is generally the same as the FEM result when the turbulence intensity is $10 \%$. However, with more than $10 \%$ of the turbulence intensity, the empirical formula may give a risky result. Then, some coefficients are introduced to modify the empirical formula according to the FEM results. The numerical examples are presented with different OCL tension classes to verify the validation of the modified empirical formula.

\section{Introduction}

The OCL is widely used in modern electrified railway systems and serves as the only source of power for electric trains [1]. As shown in Figure 1, the OCL is a tensioned cable structure constructed along the railway track, which transmits the electric current to the locomotive through the sliding contact with the pantograph installed on the carbody's roof. The quality of the current collection of the electric train is directly affected by the dynamic performance of the pantograph-OCL interaction. Typically, the OCL is the most vulnerable part of the electrified railway system, as it suffers multiple impacts from the vehicle-track vibration [2], the temperature variation [3], the irregularities in the contact line [4], and the crosswind load [5]. Often, the OCL has a large span length to reduce the construction cost and also has high flexibility to decrease the unevenness of the elasticity along the contact line. These features make the OCL very susceptible to the crosswind. Generally, wind is comprised of even wind and fluctuating wind. The former is able to cause deviation of the OCL with respect to its original configuration. The latter excites a drastic forced vibration of the OCL, which directly disturbs its contact with pantographs. More precisely, fluctuating winds may increase the fluctuation of the contact force and the possibility of the occurrence of contact loss, which creates more wear [6,7], vibration [8], fatigue [9], arcing and sparking [10, 11], and more severe accidents like dewirement and line breakage in some extreme cases. Recently, the negative effect of the wind-induced vibration of the OCL has been frequently reported from Chinese high-speed networks, especially the scarping of the pantograph head caused by the wind deviation of the contact line.

In general, the contact force between the registration strip of the pantograph and the contact line of the OCL is a direct reflection of the quality of the current collection [12]. An excessive contact force can aggravate the wear of the contact interface. In contrast, an inadequate contact force 


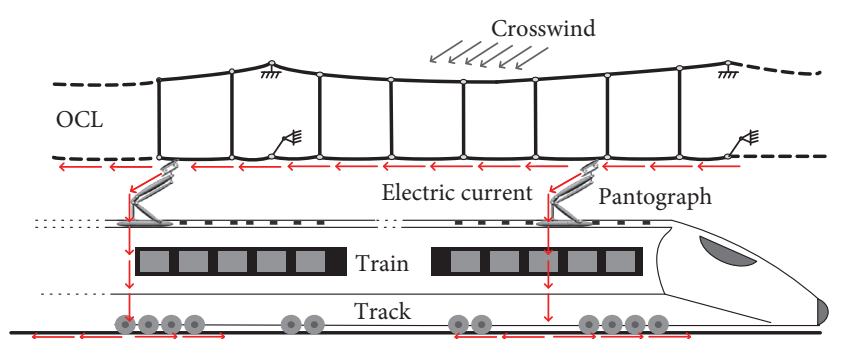

Figure 1: Schematic of a pantograph-OCL system with crosswind.

may cause the separation of the pantograph collector from the OCL. In order to ensure a stable contact force, the pantograph-OCL system should be appropriately designed. Many numerical models of the OCL have been developed to reproduce the realistic behaviour [13-15] and explore the implications of design parameters on the dynamic performance [16-18]. Based on these simulation tools, several optimisation strategies are proposed to reduce the elasticity unevenness of OCL [19], decrease the wave propagation interference [20], and avoid the resonance with pantographs [21].

The research studies mentioned above seldom consider the effect of crosswind load. Generally, there are three types of vibration for long-span structures caused by wind load. One is galloping [22], which is a kind of instability induced by negative aerodynamic damping [23]. For the OCL, galloping is rare to be observed in real life [24], except in some extreme conditions [25]. The second is vortex-induced vibration, which is common in bridge deck $[26,27]$ and power transmission line [28]. The vortex-induced vibration of OCL has not been reported in any existing literature. Apart from these two types, the buffeting of the OCL caused by fluctuating wind is the most common one, which challenges the current collection quality and the safe operation. In order to understand the aerodynamics of the OCL, the CFD model of contact line cross section was established by SanchezRebollo et al. [29] with different working conditions. In order to explore the buffeting behaviour of the OCL, Song et al. [30] derived the fluctuating forces acting on the contact line and analysed the wind-induced response of OCL based on a nonlinear finite element approach. To ensure a good interaction performance of pantograph-OCL subjected to a strong wind field, a control strategy was proposed based on the instantaneous response of the pantograph [31]. Considering the crosswind acting on the pantograph and the OCL simultaneously, Pombo et al. [32] conducted a simulation of the pantograph-OCL interaction with wind load. Then, the wind load was introduced in the simulation of multiple pantograph-OCL interactions to study its effect on the contact forces on both pantograph heads [33].

In the current industry [34], the wind deflection of the contact line is evaluated using empirical formulas, which only gives an empirical result in the design phase of the OCL. Some dynamic effects caused by the fluctuating wind, especially with high turbulence intensity, cannot be considered in the empirical equation. The work in [35] has indicated the difference in the results between the empirical equation and the response spectrum analysis. In this paper, a nonlinear finite element method is used to model the OCL. The crosswind load acting on the OCL is derived according to the fluid-induced vibration theory. The results of wind deflection evaluated by both the FEM and empirical formula are compared to indicate the shortfall of the empirical formula. Then, some modifications are suggested to improve the empirical equation.

The background and current research state are introduced in Section 1. In Section 2, the empirical formula to estimate the wind deflection is derived. In Section 3, a nonlinear finite element of the OCL is built based on FEM. In Section 4, the crosswind field along the OCL is constructed. The aerodynamic forces acting on the OCL are derived in Section 5. In Section 6, a preliminary comparative study is presented. The modification is recommended in Section 7. Some discussions and conclusions are drawn in Section 8.

\section{Estimation of Wind Deviation Using Empirical Formula}

This section presents the derivation procedure to obtain the empirical formula [34]. For the contact line, the wind load $p$ acting on the per-unit contact line can be estimated by

$$
p=0.625 V_{R}^{2} \beta_{q} \mu_{s} \mu_{z} d,
$$

in which $V_{R}^{2}$ is the design wind speed at the height of $10 \mathrm{~m}$ above ground, $\beta_{q}$ is the wind pressure non-uniformity coefficient, $\mu_{s}$ is the wind load profile coefficient, $\mu_{z}$ is the wind speed height variation coefficient, and $d$ is the diameter of the contact line.

Considering a contact line subjected to the crosswind, as shown in Figure 2, the wind deflection $y_{w}(x)$ of the contact line can be estimated by

$$
y_{w}(x)=\frac{p x(l-x)}{2 T},
$$

in which $x$ is the distance of the reference point with respect to the left steady arm, $l$ is the span length, and $T$ is the contact line tension. If no wind load is applied, the lateral displacement of the contact line with respect to the track centre can be obtained by

$$
y_{s}(x)=\frac{\left(s_{i}-s_{i+1}\right)(l-x)}{l}+s_{i+1},
$$

in which $s_{i}$ is the stagger value of the left steady arm and $s_{i+1}$ is the stagger value of the right one. Therefore, the total deviation under crosswind can be obtained by

$$
\begin{aligned}
e & =y_{w}(x)+y_{s}(x) \\
& =\frac{p x(l-x)}{2 T}+\frac{\left(s_{i}-s_{i+1}\right)(l-x)}{l}+s_{i+1} .
\end{aligned}
$$

The position of the maximum wind deflection can be estimated from equation (4) by solving the following equation: 


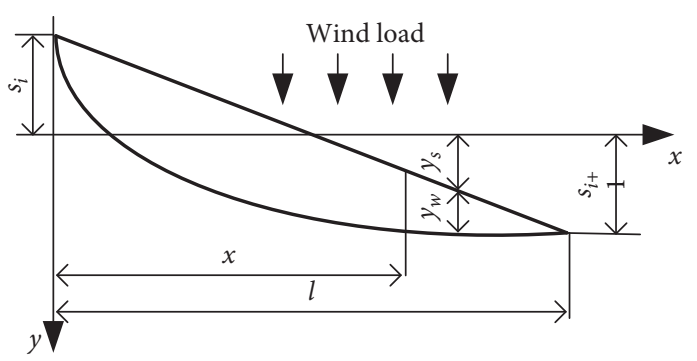

Figure 2: Derivation of contact line deflection subjected to the crosswind.

$$
\frac{\partial e}{\partial x}=0
$$

The explicit formula of the position of maximum wind deflection can be obtained as

$$
x_{\max }=\frac{l}{2}-\frac{T\left(s_{i}-s_{i+1}\right)}{p l} .
$$

Therefore, the maximum wind deflection can be obtained by substituting equation (6) into equation (4), which can be written as follows:

$$
e_{\max }=\frac{p l 2}{8 T}+\frac{T\left(s_{i}-s_{i+1}\right)^{2}}{2 p l^{2}}+\frac{s_{i}+s_{i+1}}{2} .
$$

In most cases, $s_{i}=s_{i+1}=s$. Thus, equation (7) can be simplified to

$$
e_{\max }=\frac{p l^{2}}{8 T}+\frac{2 T s^{2}}{p l^{2}}
$$

\section{Finite Element Model of OCL}

In order to describe the geometrical nonlinearity in large deformation of the OCL caused by the crosswind, a nonlinear finite element approach is adopted to model the OCL. The ANCF (absolute nodal coordinate formulation) is a commonly used nonlinear finite element method to deal with large deformations in various engineering backgrounds [36]. As shown in Figure 3, the ANCF beam is utilised to model the contact and messenger lines. The ANCF cable is employed to model the dropper and the steady arm. The claws and clamps on the dropper and steady arm are assumed as lumped masses. As for an ANCF beam element, the nodal degree of freedom (DOF) vector can be expressed by

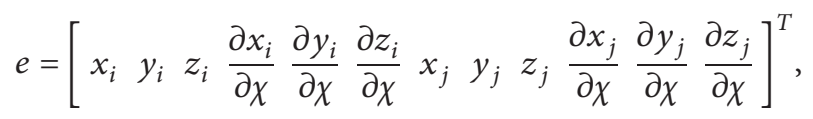

in which $\chi$ is the local longitudinal coordinate in the undeformed configuration from 0 to the element length $L_{0}$. The position vector in the deformed configuration $\mathbf{r}$ can be interpolated using the shape function matrix $\mathbf{S}$ as

$$
\mathbf{r}=\text { Se }
$$

According to [37], $\mathbf{S}$ is defined using a classic form of shape function for beam element as follows:

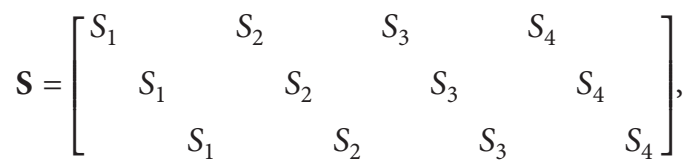

$$
\begin{aligned}
& S_{1}(\xi)=1-3 \xi^{2}+2 \xi^{3}, \\
& S_{2}(\xi)=l_{0}\left(\xi+\xi^{3}-2 \xi^{2}\right) \text {, } \\
& S_{3}(\xi)=3 \xi^{2}-2 \xi^{3} \text {, } \\
& S_{4}(\xi)=l_{0}\left(\xi^{3}-\xi^{2}\right) \text {. }
\end{aligned}
$$

The strain energy of the beam element can be calculated by the summation of the energies in axial and bending deformation as

$$
U=\frac{1}{2} \int_{0}^{L_{0}}\left(E A \varepsilon_{l}^{2}+E I \kappa^{2}\right) \mathrm{d} \chi
$$

in which $E$ is the elastic modulus, $A$ is the section area, $I$ is the inertial moment of the beam, $\varepsilon_{l}$ is the longitudinal strain, and $\kappa$ is the curvature. Differentiating equation (12) with respect to the element DOF vector e yields the generalised elastic force vector $\mathbf{Q}$ as

$$
\mathbf{Q}=\left(\frac{\partial U}{\partial \mathbf{e}}\right)^{T}=\mathbf{K}_{e} \mathbf{e}
$$

The secant stiffness matrix $\mathbf{K}_{\mathrm{e}}$ for the given element can be determined according to equation (13). In an integration algorithm, the tangent stiffness matrix $\mathbf{K}_{\mathrm{T}}$ is mostly used to calculate the incremental nodal DOF vector $\Delta \mathbf{e}$. Notably, in the shape-finding procedure, the tangent stiffness matrix $\mathbf{K}_{\mathrm{L}}$ related to the incremental unstrained length $\Delta L_{0}$ is necessary to determine the unstrained size of each element. Thus, the tangent stiffness matrices $\mathbf{K}_{\mathrm{T}}$ and $\mathbf{K}_{\mathrm{L}}$ are obtained by differentiating both sides of equation (13).

$$
\Delta \mathbf{F}=\frac{\partial \mathbf{Q}}{\partial \mathbf{e}} \Delta \mathbf{e}+\frac{\partial \mathbf{Q}}{\partial L_{0}} \Delta L_{0}=\mathbf{K}_{T} \Delta \mathbf{e}+\mathbf{K}_{L} \Delta L_{0} .
$$

The stiffness matrix of the ANCF cable element can be obtained through a similar derivation procedure. It should be noted that the cable element used to describe the nonlinear behaviour of droppers cannot withstand compression. The axial stiffness is set to zero when the dropper works in compression. Assembling the element matrices by the FEM yields the global incremental equilibrium equation for the whole OCL as follows:

$$
\Delta \mathbf{F}^{G}=\mathbf{K}_{T}^{G} \Delta \mathbf{U}_{C}+\mathbf{K}_{L}^{G} \Delta \mathbf{L}_{0},
$$

where $\Delta \mathbf{F}^{G}$ is the global unbalanced force vector and $\mathbf{K}_{T}^{G}$ and $\mathbf{K}_{L}^{G}$ are the global stiffness matrices related to the incremental nodal displacement vector $\Delta \mathbf{U}_{C}$ and the incremental unstrained length vector $\Delta \mathbf{L}_{0}$, respectively. Additional constraint 


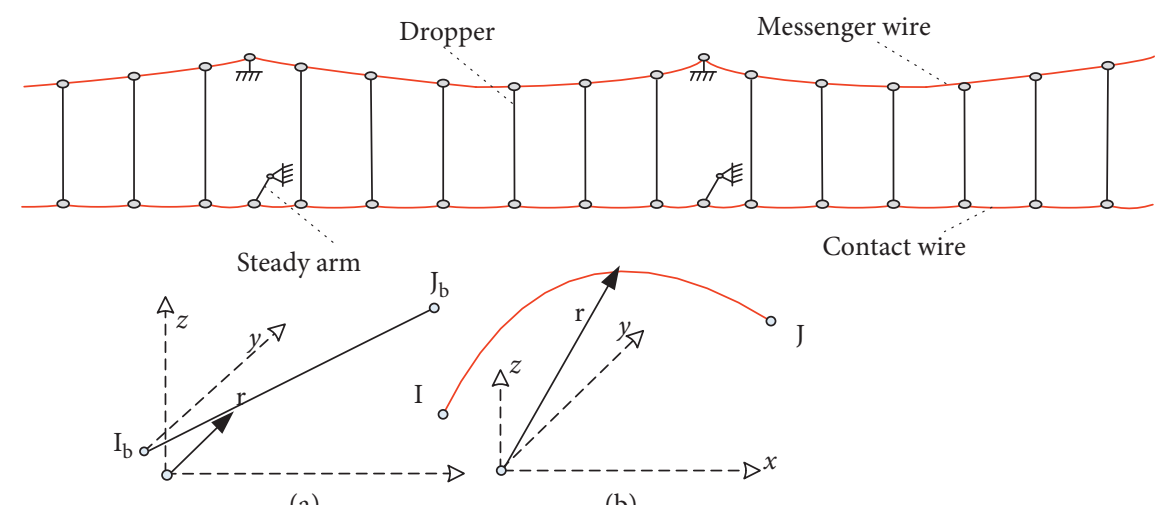

(a)

(b)

FIgURE 3: Finite element model of the OCL. (a) ANCF cable. (b) ANCF beam.

conditions for the design specification can be provided to reduce the number of unknowns in equation (15) and ensure a unique solution. Then, the Newton-Raphson iteration can be used to solve the equilibrium state of the OCL with the gravity and tensions [38]. Including a consistent mass matrix $\mathbf{M}_{C}^{G}$ and a Rayleigh damping matrix $\mathbf{C}_{C}^{G}$, the equation of motion for the OCL is written by

$$
\mathbf{M}_{C}^{G} \ddot{U}_{C}(t)+\mathbf{C}_{C}^{G} \dot{U}_{C}(t)+\mathbf{K}_{C}^{G}(t) \mathbf{U}_{C}(t)=\mathbf{F}_{C}^{G}(t),
$$

in which $\mathbf{K}_{C}^{G}(t)$ is the global stiffness matrix of the OCL and $\mathbf{F}_{C}^{G}(t)$ is the external force vector applied on the OCL. Table 1 presents the main parameters of a conventional-speed railway OCL. The initial configuration of the OCL can be calculated according to the above approach, of which the central two spans are taken as the analysis object in the following analysis.

\section{Crosswind Field of OCL}

Generally, the natural wind can be divided into even wind and fluctuating wind. For a given spatial point $M$, the wind speed at this point can be expressed by

$$
V(M, t)=\tilde{V}(M, t)+V^{\prime}(M, t)
$$

in which $\widetilde{V}(M, t)$ is the even wind speed and $V^{\prime}(M, t)$ is the fluctuating wind speed. They can be expressed by the following two vectors:

$$
\begin{aligned}
\widetilde{V}(M) & =\left[\begin{array}{lll}
U(M) & 0 & 0
\end{array}\right]^{T}, \\
V^{\prime}(M, t) & =\left[\begin{array}{lll}
u(M, t) & w(M, t) & v(M, t)
\end{array}\right]^{T},
\end{aligned}
$$

in which $U(M)$ is the steady wind speed in the longitudinal direction and $u(M, t), w(M, t)$, and $v(M, t)$ denote the fluctuating wind speed in the longitudinal, lateral, and vertical directions, respectively. In this section, the spatial wind field is constructed for the OCL.

The empirical spectrum is a standard way to describe the stochastics of the fluctuating wind speed. In this work, the von Karman spectrums [39] in longitudinal, lateral, and vertical directions are adopted to generate the wind speed time
TABLE 1: OCL property parameters.

\begin{tabular}{lc}
\hline Span length & $60 \mathrm{~m}$ \\
Contact line tension & $13 \mathrm{kN}$ \\
Messenger line tension & $13 \mathrm{kN}$ \\
Contact line area & $120 \mathrm{~mm}^{2}$ \\
Messenger line area & $120 \mathrm{~mm}^{2}$ \\
Contact line linear density & $1.07 \mathrm{~kg} / \mathrm{m}$ \\
Messenger line linear density & $1.06 \mathrm{~kg} / \mathrm{m}$ \\
\hline
\end{tabular}

histories. For two arbitrary spatial points $M$ and $P$, the power spectral density matrix for this point can be expressed by

$$
\mathbf{S}_{V}^{M, P}(\omega)=\left[\begin{array}{ccc}
\sqrt{S_{u}^{M} S_{u}^{P}} & 0 & \operatorname{coh}_{u v}^{M, P} \sqrt{S_{u}^{M} S_{v}^{P}} \\
0 & \sqrt{S_{w}^{M} S_{w}^{P}} & 0 \\
\operatorname{coh}_{v u}^{M, P} \sqrt{S_{v}^{M} S_{u}^{P}} & 0 & \sqrt{S_{v}^{M} S_{v}^{P}}
\end{array}\right] \text {, }
$$

in which $\operatorname{coh}_{\varepsilon \eta}^{M, P}$ represents the correlation function between two points $M$ and $P$ in $\varepsilon$ and $\eta(\varepsilon, \eta=u, w, v)$ directions. The power spectral density matrix for one point $M$ can be expressed by

$$
\mathbf{S}_{V}^{M}(\omega)=\left[\begin{array}{ccc}
S_{u}^{M} & 0 & \operatorname{coh}_{u v}^{M, M} \sqrt{S_{u}^{M} S_{v}^{M}} \\
0 & S_{w}^{M} & 0 \\
\operatorname{coh}_{v u}^{M, M} \sqrt{S_{v}^{M} S_{u}^{M}} & 0 & S_{v}^{M}
\end{array}\right]
$$

After determining an appropriate spatial grid, the global power spectral density matrix $\mathbf{S}_{V}(\omega)$ can be obtained by assembling equations (19) and (20). The spatial grid depicted in Figure 4 is adopted. It is seen that the spatial grid has two layers in the vertical direction. The top one is for the messenger/upper half of the dropper line. The second one is for the contact/lower half of the dropper line. The spatial interval of the grid in the $X$ direction is $10 \mathrm{~m}$. After obtaining the spectrum matrix, the time history of fluctuating wind speed can be obtained through the inverse Fourier transform. 


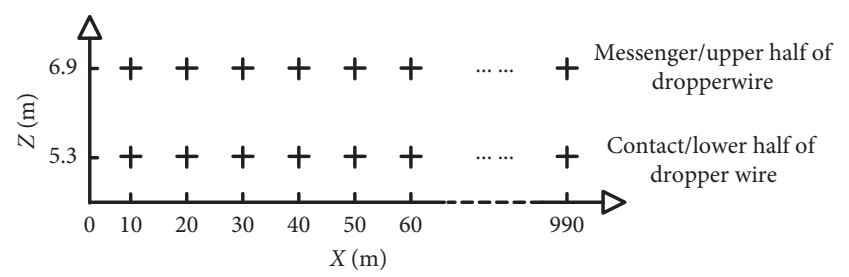

FIGURE 4: Spatial grid for the OCL.

\section{Aerodynamic Forces}

After obtaining the fluctuating wind speed, the aerodynamic forces are derived in this section. As shown in Figure 5, the aerodynamic forces acting on the contact line are derived as follows. The aerodynamic forces on the messenger line, dropper, and steady arm can be obtained through a similar derivation procedure. The contact line has a certain displacement in the lateral direction, and the contact line is not always perpendicular to $U$. Therefore, the wind speed vectors should be converted to the local reference system for each element. For each element, the even wind $U^{e}$ and fluctuating wind components $u^{e}, v^{e}$, and $w^{e}$ in the element local reference system can be obtained by the spatial coordinate transformation [40]. The contact line cross section subjected to $U^{e}, u^{e}$, and $w^{e}$ is shown in Figure 5. As $U^{e}$ is parallel to the $y$-axis, " $y-o-z$ " is called an absolute wind-axis reference system. The drag $F_{D}^{e}$ and lift $F_{L}^{e}$ are the aerodynamic forces acting on the contact line section caused by the wind load. A dynamic wind angle $\beta$ is induced by the movement of the contact line subjected to the wind load, which can change the angle of attack. $\beta$ can be estimated by

$$
\beta=\arctan \left(\frac{w^{e}-\dot{z}_{c r}^{e}}{U^{e}+u^{e}-\dot{y}_{c r}^{e}}\right),
$$

in which $\dot{z}_{c r}^{e}$ and $\dot{y}_{c r}^{e}$ are the vertical and lateral velocities of the contact line in the reference system (called relative windaxis reference system) defined by the dynamic angle $b$, lift $F_{L r}^{e}$, and drag $F_{D r}^{e} . \dot{z}_{c r}^{e}$ and $\dot{y}_{c r}^{e}$ can be obtained by transferring the vertical and lateral velocities $\dot{z}_{c}^{e}$ and $\dot{y}_{c}^{e}$ in the absolute wind-axis reference system to relative wind-axis reference system. $F_{L r}^{e}$ and $F_{D r}^{e}$ can be expressed by

$$
\left\{\begin{array}{l}
F_{L r}^{e}=\frac{1}{2} \rho_{\text {air }} U_{r}^{e} L_{e} D C_{L}(\beta), \\
F_{D r}^{e}=\frac{1}{2} \rho_{\text {air }} U_{r}^{e} L_{e} D C_{D}(\beta),
\end{array}\right.
$$

in which $\rho_{\text {air }}$ is the air density, $D$ is the diameter of the contact line cross section, $C_{L}(\beta)$ and $C_{D}(\beta)$ are the lift and drag coefficients at the angle of attack $\beta$, and $U_{r}^{e}$ is the effective wind velocity, which can be expressed by

$$
U_{r}^{e}=\sqrt{\left(w^{e}-\dot{z}_{c r}^{e}\right)^{2}+\left(U^{e}+v^{e}-\dot{y}_{c r}^{e}\right)^{2}} .
$$

According to the geometrical relationship, the drag $F_{D}^{e}$ and lift $F_{L}^{e}$ in the absolute wind-axis coordinate system can be obtained by

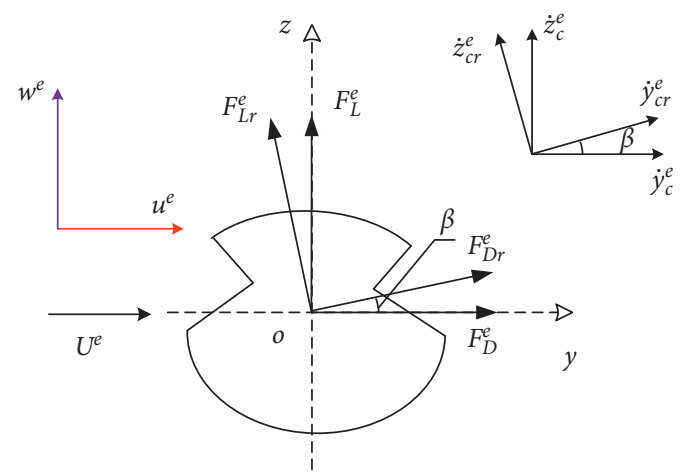

Figure 5: Derivation of aerodynamic forces acting on the contact line cross section.

$$
\left\{\begin{array}{l}
F_{D}^{e}=F_{D r}^{e} \cos (\beta)-F_{L r}^{e} \sin (\beta), \\
F_{L}^{e}=F_{D r}^{e} \sin (\beta)+F_{L r}^{e} \cos (\beta) .
\end{array}\right.
$$

The spatial coordinate transformation is performed again to transfer $F_{D}^{e}$ and $F_{L}^{e}$ to the global reference system, which can be directly exerted on the finite element model.

The aerodynamic coefficients $C_{\mathrm{D}}$ and $C_{\mathrm{L}}$ usually are dependant on the cross-sectional profile and the Reynolds number. In this section, the wind tunnel test results obtained in the authors' previous work [41] are adopted in the following simulations.

\section{Comparative Analysis}

According to the technical specification, the design wind speed for the railway OCL is $30 \mathrm{~m} / \mathrm{s}$, which is defined in the following analyses. The numerical simulations of wind deflection with different turbulence intensities from $9 \%$ to $25 \%$ are performed. Taking the positions where the maximum wind deflection occurs as an analysis object, Figure 6 shows maximum wind deflection with different turbulence intensities. The red dash line denotes the result given by equation (8). The blue dash line represents the maximum allowed deflection specified in the standard [42]. It is seen that the increase of the turbulence intensity significantly increases the maximum wind deflection. However, the variation of the wind deflection versus the turbulence intensity cannot be considered in the empirical formula. The result given by the empirical formula is generally the same as the FEM result with 10\% turbulence intensity. For the turbulence intensity smaller than $10 \%$, the empirical equation only gives conservative results. In contrast, when the turbulence intensity is bigger than $10 \%$, the empirical formula may give dangerous results. Mainly, when the turbulence intensity is over $15 \%$, the maximum wind deflection is over the safety threshold, which may result in fatal accidents. Figures 7 and 8 present the time history of the contact line wind deflection in the position where the maximum wind deflection occurs with the $9 \%$ and $11 \%$ turbulence intensities, respectively. It is seen that all the displacements with $9 \%$ turbulence intensity are smaller than 


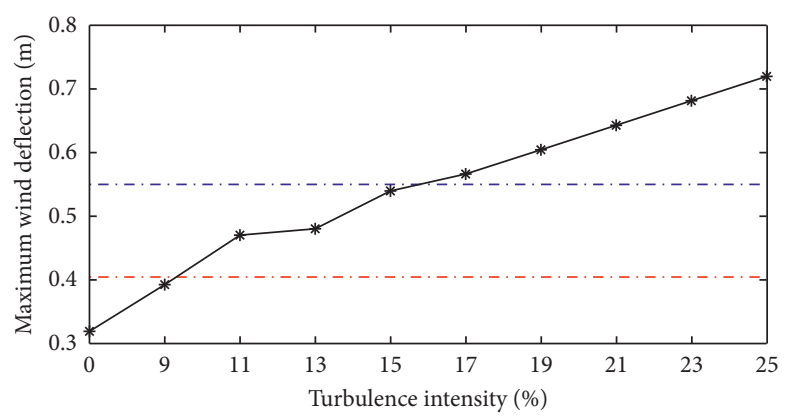

FIGURE 6: Maximum wind deflection with different turbulence intensities.

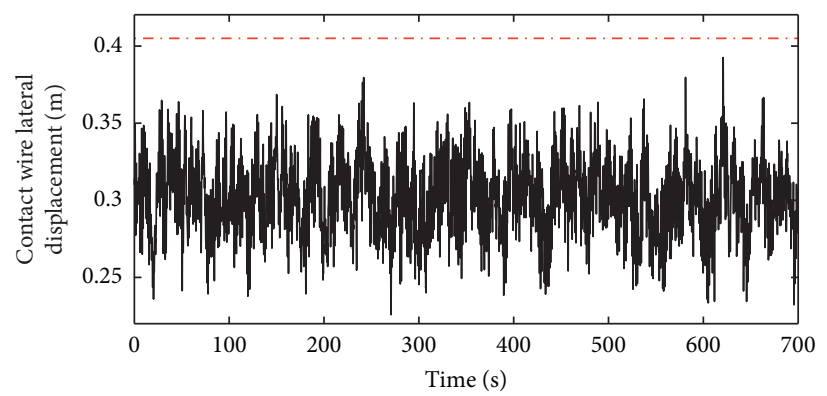

FIgURE 7: Lateral wind deflection with $9 \%$ turbulence intensity.

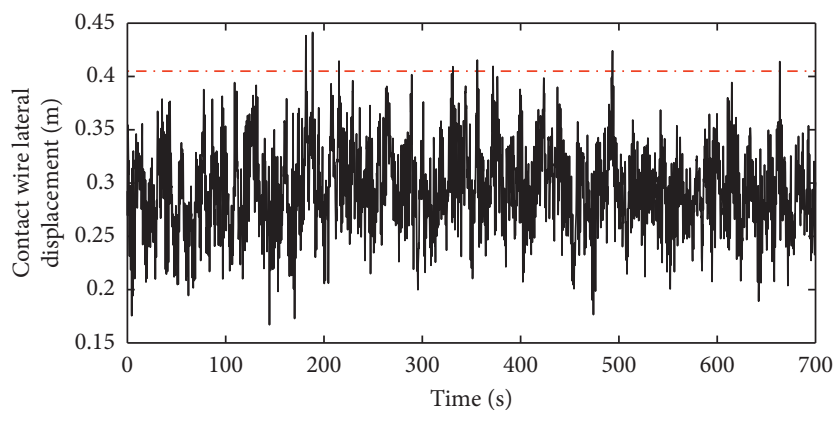

Figure 8: Lateral wind deflection with $11 \%$ turbulence intensity.

the value given by the empirical formula. Some outliers can be seen when the turbulence intensity moves up to $11 \%$.

Assuming that the contact line wind deflection follows the normal distribution, the probability density functions (PDFs) of contact line displacement at the maximum deflection position are presented in Figure 9. It is seen that the contact line displacement has a $0 \%$ possibility to exceed the empirical value with $9 \%$ turbulence intensity. However, this possibility increases to $0.19 \%, 0.88 \%, 2.15 \%$, and $3.31 \%$ with the turbulence intensities of $11 \%, 13 \%, 15 \%$, and $17 \%$. With $25 \%$ turbulence intensity, the contact line displacement has a 9.65\% possibility to exceed the empirical value, which will definitely give risky results.

\section{Modification of Empirical Formula}

In this section, a modified empirical formula is proposed to make it consider the change of the turbulence intensity. The modified form of the empirical formula is given by introducing the modification coefficients $\alpha_{1}, \alpha_{2}, \alpha_{3}$, and $\alpha_{4}$ as follows:

$$
e_{\max }=\frac{p l^{2}}{8 T}\left(\alpha_{1} I^{3}+\alpha_{2} I^{2}+\alpha_{3} I+\alpha_{4}\right)+\frac{2 T s^{2}}{p l^{2}},
$$

in which $I$ represents the turbulence intensity $\times 100$. Using the simulation results in Figure 6 , the modified coefficients $\alpha_{1}, \alpha_{2}, \alpha_{3}$, and $\alpha_{4}$ can be obtained by the curve fitting, which is indicated in Figure 10. The fitted coefficients $\alpha_{1}, \alpha_{2}, \alpha_{3}$, and $\alpha_{4}$ are presented in Table 2 .

In order to validate the proposed modified empirical formula, several simulations with different OCL systems are performed with $15 \%$ turbulence intensity. In these simulations, the main parameter of the OCL, tension, is changed to be $0.8-2$ times the original value. The resulting maximum wind deflections evaluated by the FEM, traditional empirical formula, and modified empirical formula are presented in Figure 11. It is seen that the accuracy of the empirical 


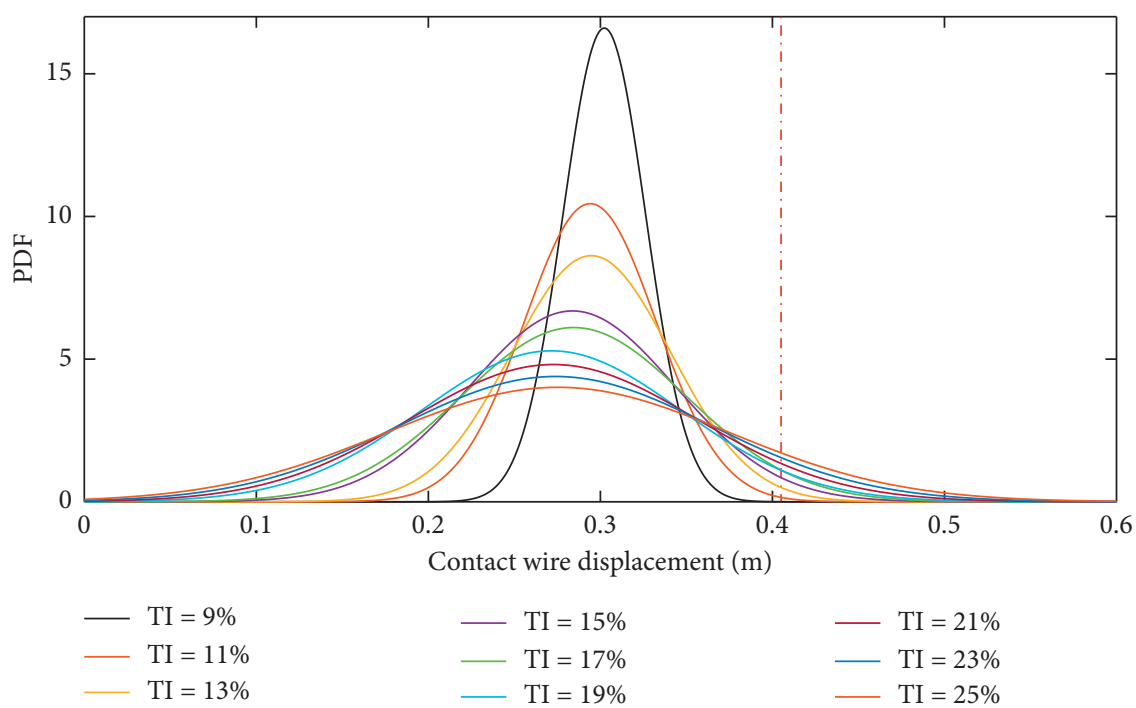

Figure 9: PDF of contact line displacement with different turbulence intensities.

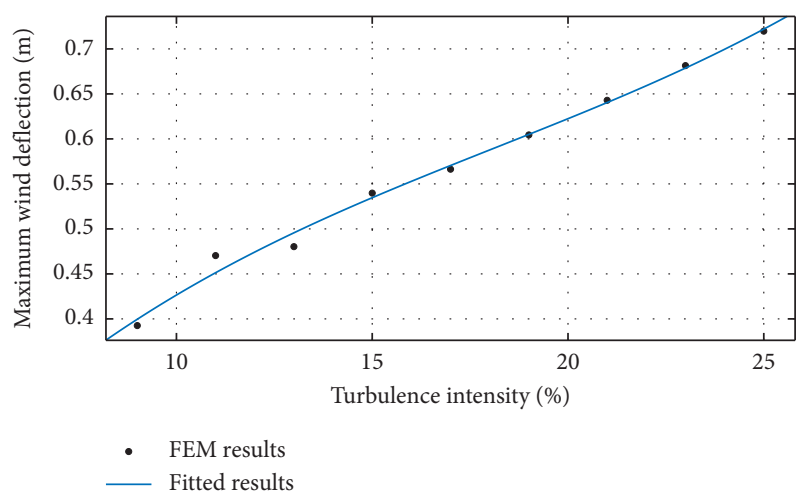

FIgURE 10: Curve fitting of modified empirical formula.

TABLE 2: Fitted coefficients in the modified empirical formula.

\begin{tabular}{lccc}
\hline$\alpha_{1}$ & $\alpha_{2}$ & $\alpha_{3}$ & $\alpha_{4}$ \\
\hline 0.0001267 & -0.006906 & 0.1765 & -0.136 \\
\hline
\end{tabular}

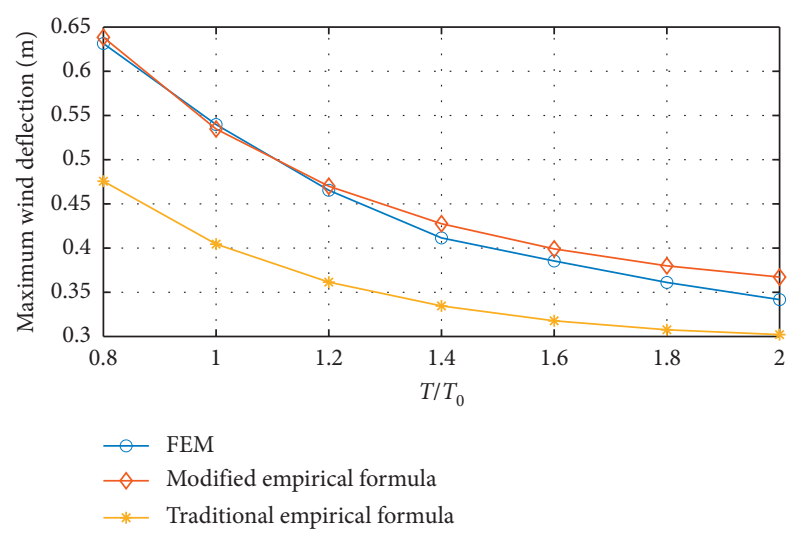

Figure 11: Maximum wind deflection with different tension classes calculated by the FEM and modified and traditional empirical formulas. formula to estimate the maximum wind deflection is significantly improved after the modification. For most cases, the evaluated results of the modified empirical formula are slightly larger than the FEM results, which demonstrates that the modified empirical formula gives conservative results in the design phase to ensure operational safety.

\section{Conclusions}

In this paper, a comparative study of the railway OCL wind deflection is performed based on the traditional empirical formula and FEM. A nonlinear finite element approach is presented to model the OCL. The aerodynamic forces acting on the OCL caused by the crosswind load are derived. The simulation results indicate that the empirical formula result can only be effective when the turbulence intensity is $10 \%$. Risky results may be given by the empirical formula with a turbulence intensity of more than $10 \%$. The empirical formula is modified by introducing some modification coefficients. According to the FEM results, a modified empirical formula is obtained. Some numerical examples are presented with different tension classes to verify the validation of the present modified empirical formula.

\section{Data Availability}

No data were used to support this study.

\section{Conflicts of Interest}

The authors declare that they have no conflicts of interest.

\section{Acknowledgments}

This study was supported in part by the National Natural Science Foundation of China (U1734202) and the funding of Chengdu Guojia Electrical Engineering Co. Ltd. (no. NEEC2019-B09). 


\section{References}

[1] W. Zhang, D. Zou, M. Tan, N. Zhou, R. Li, and G. Mei, "Review of pantograph and catenary interaction," Frontiers of Mechanical Engineering, vol. 13, no. 2, pp. 311-322, 2018.

[2] Y. Song, Z. Wang, Z. Liu, and R. Wang, "A spatial coupling model to study dynamic performance of pantograph-catenary with vehicle-track excitation," Mechanical Systems and Signal Processing, vol. 151, Article ID 107336, 2021.

[3] A. Landi, L. Menconi, and L. Sani, "Hough transform and thermo-vision for monitoring pantograph-catenary system," Proceedings of the Institution of Mechanical Engineers-Part F: Journal of Rail and Rapid Transit, vol. 220, no. 4, pp. 435-447, 2006.

[4] Y. Song, Z. Z. Liu, A. Ronnquist, P. Navik, and Z. Z. Liu, "Contact wire irregularity stochastics and effect on high-speed railway pantograph-catenary interactions," IEEE Transactions on Instrumentation and Measurement, vol. 69, pp. 8196-8206, 2020.

[5] D. Liu, Z. Lu, T. Cao, L. Zhang, and C. Zhu, "Experimental study on vibration displacement of a CRH2 EMU under strong wind conditions," in Proceedings of the 2016 IEEE International Conference on Intelligent Rail Transportation ICIRT, pp. 377-381, IEEE, Birmingham, UK, August 2016.

[6] A. W. C. Shing and P. P. L. Wong, "Wear of pantograph collector strips," Proceedings of the Institution of Mechanical Engineers-Part F: Journal of Rail and Rapid Transit, vol. 222, no. 2, pp. 169-176, 2008.

[7] G. Bucca and A. Collina, "A procedure for the wear prediction of collector strip and contact wire in pantograph-catenary system," Wear, vol. 266, no. 1-2, pp. 46-59, 2009.

[8] P. Boffi, G. Cattaneo, L. Amoriello et al., "Optical fiber sensors to measure collector performance in the pantograph-catenary interaction," IEEE Sensors Journal, vol. 9, no. 6, pp. 635-640, 2009.

[9] J. P. Massat, T. M. L. Nguyen-Tajan, H. Maitournam, and E. Balmès, "Fatigue analysis of catenary contact wires for high speed trains," in Proceedings of the 9th World Congress Railway Research, Paris, France, May 2011.

[10] M. Karakose, O. Yaman, I. Aydin, and E. Karakose, "Real-time condition monitoring approach of pantograph-catenary system using FPGA," in Proceedings of the IEEE International Conference on Industrial Informatics, pp. 481-486, Poitiers, France, July 2016.

[11] I. Aydin, M. Karakose, and E. Akin, "Anomaly detection using a modified kernel-based tracking in the pantograph-catenary system," Expert Systems with Applications, vol. 42, no. 2, pp. 938-948, 2015.

[12] J. Zhang, W. Liu, and Z. Zhang, "Study on characteristics location of pantograph-catenary contact force signal based on wavelet transform," IEEE Transactions on Instrumentation and Measurement, vol. 68, no. 2, pp. 402-411, 2019.

[13] S. Bruni, J. Ambrosio, A. Carnicero et al., "The results of the pantograph-catenary interaction benchmark," Vehicle System Dynamics, vol. 53, no. 3, pp. 412-435, 2015.

[14] S. H. Kia, F. Bartolini, A. Mpanda-Mabwe, and R. Ceschi, "Pantograph-catenary interaction model comparison," in Proceedings of the IECON 2010-36th Annual Conference on IEEE Industrial Electronics Society, pp. 1584-1589, Glendale, AZ, USA, November 2010.

[15] Y. Song, P. Antunes, J. Pombo, and Z. Liu, "A methodology to study high-speed pantograph-catenary interaction with realistic contact wire irregularities," Mechanism and Machine Theory, vol. 152, Article ID 103940, 2020.
[16] T.-J. Park, C.-S. Han, and J.-H. Jang, "Dynamic sensitivity analysis for the pantograph of a high-speed rail vehicle," Journal of Sound and Vibration, vol. 266, no. 2, pp. 235-260, 2003.

[17] S. Gregori, M. Tur, J. E. Tarancón, and F. J. Fuenmayor, "Stochastic Monte Carlo simulations of the pantograph-catenary dynamic interaction to allow for uncertainties introduced during catenary installation," Vehicle System Dynamics, vol. 57, no. 4, pp. 471-492, 2019.

[18] J. Zhang, W. Liu, and Z. Zhang, "Sensitivity analysis and research on optimisation methods of design parameters of high-speed railway catenary," IET Electrical Systems in Transportation, vol. 9, no. 3, pp. 150-156, 2019.

[19] S. Gregori, M. Tur, E. Nadal, and F. J. Fuenmayor, “An approach to geometric optimisation of railway catenaries," Vehicle System Dynamics, vol. 56, no. 8, pp. 1162-1186, 2018.

[20] Z. Xu, Y. Song, and Z. Liu, "Effective measures to improve current collection quality for double pantographs and catenary based on wave propagation analysis," IEEE Transactions on Vehicular Technology, vol. 69, no. 6, pp. 6299-6309, 2020.

[21] Y. Song, Z. Liu, F. Duan, Z. Xu, and X. Lu, "Wave propagation analysis in high-speed railway catenary system subjected to a moving pantograph," Applied Mathematical Modelling, vol. 59, pp. 20-38, 2018.

[22] J. Si, X. Rui, B. Liu, L. Zhou, and S. Liu, "Study on a new combined anti-galloping device for UHV overhead transmission lines," IEEE Transactions on Power Delivery, vol. 34, no. 6, pp. 2070-2078, 2019.

[23] M. Cai, B. Yan, X. Lu, and L. Zhou, "Numerical simulation of aerodynamic coefficients of iced-quad bundle conductors," IEEE Transactions on Power Delivery, vol. 30, no. 4, pp. 1669-1676, 2015.

[24] Y. Song, Z. Liu, H. Wang, J. Zhang, X. Lu, and F. Duan, "Analysis of the galloping behaviour of an electrified railway overhead contact line using the non-linear finite element method," Proceedings of the Institution of Mechanical Engineers-Part F: Journal of Rail and Rapid Transit, vol. 232, no. 10, pp. 2339-2352, 2018.

[25] S. Avila-Sanchez, O. Lopez-Garcia, A. Cuerva, and J. Meseguer, "Assesment of the transverse galloping stability of a railway overhead located above a railway bridge," International Journal of Mechanical Sciences, vol. 131-132, pp. 649-662, 2017.

[26] M. Zhang, F. Xu, and Y. Han, “Assessment of wind-induced nonlinear post-critical performance of bridge decks," Journal of Wind Engineering and Industrial Aerodynamics, vol. 203, 2020.

[27] M. Zhang, F. Xu, and O. Øiseth, “Aerodynamic damping models for vortex-induced vibration of a rectangular 4:1 cylinder: comparison of modeling schemes," Journal of Wind Engineering and Industrial Aerodynamics, vol. 205, 2020.

[28] M. Kraus and P. Hagedorn, "Aeolian vibrations: wind energy input evaluated from measurements on an energized transmission line," IEEE Transactions on Power Delivery, vol. 6, 1991.

[29] C. Sanchez-Rebollo, E. Velez, and J. R. Jimenez-Octavio, "Numerical characterization of real railway overhead cables," Wind and Structures, vol. 21, no. 1, pp. 105-117, 2015.

[30] Y. Song, Z. Liu, H. Wang, X. Lu, and J. Zhang, "Nonlinear analysis of wind-induced vibration of high-speed railway catenary and its influence on pantograph-catenary interaction," Vehicle System Dynamics, vol. 54, no. 6, pp. 723-747, 2016. 
[31] Y. Song, Z. Liu, H. Ouyang, H. Wang, and X. Lu, "Sliding mode control with PD sliding surface for high-speed railway pantograph-catenary contact force under strong stochastic wind field," Shock and Vibration, vol. 2017, Article ID 4895321, 16 pages, 2017.

[32] J. Pombo, J. Ambrósio, M. Pereira, F. Rauter, A. Collina, and A. Facchinetti, "Influence of the aerodynamic forces on the pantograph-catenary system for high-speed trains," Vehicle System Dynamics, vol. 47, no. 11, pp. 1327-1347, 2009.

[33] J. Pombo and J. Ambrósio, "Environmental and track perturbations on multiple pantograph interaction with catenaries in high-speed trains," Computers \& Structures, vol. 124, pp. 88-101, 2013.

[34] J. Wu, Pantograph and Contact Line System, Elsevier, Amsterdam, Netherlands, 2017.

[35] Y. Song, M. Zhang, and H. Wang, "A response spectrum analysis of wind deflection in railway overhead contact lines using pseudo-excitation method," IEEE Transactions on Vehicular Technology, vol. 70, 2021.

[36] B. Zemljarič and V. Ažbe, "Analytically derived matrix endform elastic-forces equations for a low-order cable element using the absolute nodal coordinate formulation," Journal of Sound and Vibration, vol. 446, pp. 263-272, 2019.

[37] K. J. Bathe, Finite Element Procedures, Prentice Hall, Hoboken, NJ, USA, 2005.

[38] Y. Song, Z. Liu, Z. Xu, and J. Zhang, "Developed moving mesh method for high-speed railway pantograph-catenary interaction based on nonlinear finite element procedure," International Journal of Reality Therapy, vol. 7, no. 3, pp. 173-190, 2019.

[39] E. E. Morfiadakis, G. L. Glinou, and M. J. Koulouvari, “The suitability of the von Karman spectrum for the structure of turbulence in a complex terrain wind farm," Journal of Wind Engineering and Industrial Aerodynamics, vol. 62, 1996.

[40] Y. Song, Z. Liu, F. Duan, X. Lu, and H. Wang, "Study on windinduced vibration behavior of railway catenary in spatial stochastic wind field based on nonlinear finite element procedure," Journal of Vibration Acoustics Transactions ASME.vol. 140, pp. 1-14, Article ID 011010, 2018.

[41] Y. C. Liu, Z. G. Liu, Y. Song, and Z. W. Han, "Simulation calculation and wind tunnel test of static aerodynamic parameters of high-speed railway contact line," Tiedao Xuebao/ Journal China Railway Society, vol. 36, pp. 33-38, 2014.

[42] European Committee for Electrotechnical Standardization EN 50367, Railway Applications-Current Collection Systems-Technical Criteria for the Interaction between Pantograph and Overhead Line, European Standards (EN), Brussels, Belgium, 2016. 